\title{
Diagnostic properties of nerve conduction tests in population-based
} carpal tunnel syndrome

\author{
Isam Atroshi*1, Christina Gummesson², Ragnar Johnsson ${ }^{3}$ and \\ Ewald Ornstein 4
}

Address: ${ }^{1}$ Department of Orthopedics, Hässleholm-Kristianstad Hospitals, Kristianstad, Sweden, ${ }^{2}$ Department of Physical Therapy, Lund University, Lund, Sweden, ${ }^{3}$ Department of Orthopedics, Lund University Hospital, Lund, Sweden and ${ }^{4}$ Department of Orthopedics, HässleholmKristianstad Hospitals, Hässleholm, Sweden

Email: Isam Atroshi* - isam.atroshi@mailbox.swipnet.se; Christina Gummesson - christina.gummesson@sjukgym.lu.se; Ragnar Johnsson - ragnar.johnsson@ort.lu.se; Ewald Ornstein - ewald.ornstein@skane.se

* Corresponding author

Published: 7 May 2003

BMC Musculoskeletal Disorders 2003, 4:9

This article is available from: http://www.biomedcentral.com/I47/-2474/4/9 media for any purpose, provided this notice is preserved along with the article's original URL.

Received: 24 February 2003 Accepted: 7 May 2003

(C) 2003 Atroshi et al; licensee BioMed Central Ltd. This is an Open Access article: verbatim copying and redistribution of this article are permitted in all

\begin{abstract}
Background: Numerous nerve conduction tests are used for the electrodiagnosis of carpal tunnel syndrome (CTS), with a wide range of sensitivity and specificity reported for each test in clinical studies. The tests have not been assessed in population-based studies. Such information would be important when using electrodiagnosis in epidemiologic research. The purpose of this study was to compare the diagnostic accuracy of various nerve conduction tests in population-based CTS and determine the properties of the most accurate test.
\end{abstract}

Methods: In a population-based study a questionnaire was mailed to a random sample of 3,000 persons. Of 2,466 responders, 262 symptomatic (numbness/tingling in the radial fingers) and 125 randomly selected asymptomatic responders underwent clinical and electrophysiologic examinations. A standardized hand diagram was administered to the symptomatic persons. At the clinical examination, the examining surgeon identified 94 symptomatic persons as having clinically certain CTS. Nerve conduction tests were then performed on the symptomatic and the asymptomatic persons by blinded examiners. Analysis with receiver operating characteristic (ROC) curves was used to compare the diagnostic accuracy of the nerve conduction tests in distinguishing the persons with clinically certain CTS from the asymptomatic persons.

Results: No difference was shown in the diagnostic accuracy of median nerve distal motor latency, digit-wrist sensory latency, wrist-palm sensory conduction velocity, and wrist-palm/forearm sensory conduction velocity ratio (area under curve, $0.75-0.76$ ). Median-ulnar digit-wrist sensory latency difference had a significantly higher diagnostic accuracy (area under curve, 0.80 ). Using the optimal cutoff value of $0.8 \mathrm{~ms}$ for abnormal sensory latency difference shown on the ROC curve the sensitivity was $70 \%$, specificity $82 \%$, positive predictive value $19 \%$ and negative predictive value $98 \%$. Based on the clinical diagnosis among the symptomatic persons, the hand diagram (classified as classic/probable or possible/unlikely CTS) had high sensitivity but poor specificity.

Conclusions: Using the clinical diagnosis of CTS as the criterion standard, nerve conduction tests had moderate sensitivity and specificity and a low positive predictive value in population-based CTS. Measurement of median-ulnar sensory latency difference had the highest diagnostic accuracy. The performance of nerve conduction tests in population-based CTS does not necessarily apply to their performance in clinical settings. 


\section{Background}

Nerve conduction tests are commonly used in the assessment of patients with numbness, tingling and pain in the hands. Carpal tunnel syndrome (CTS) is one of the most common disorders for which nerve conduction tests are performed. A variety of median nerve motor and sensory tests have been introduced for the purpose of establishing the presence of median neuropathy in patients with CTS [1]. Previous publications involving the electrodiagnosis of CTS have reported a wide range of results for the sensitivity of median nerve distal motor latency (29\% to $81 \%$ ), wrist-digit sensory latency ( $44 \%$ to $100 \%)$, and wristpalm sensory conduction velocity ( $45 \%$ to $100 \%$ ), and of median-ulnar sensory latency difference (57\% to $100 \%$ ) [2]. All these reports involved referred patients; no population-based studies have assessed the performance of various nerve conduction tests in CTS. Such information would be important if electrodiagnosis is to be used in epidemiologic research.

Practice parameter for electrodiagnosis in CTS has been published [3] and recently slightly modified [4,5]. The proposed standards are measurement of median nerve wrist-digit sensory conduction and, when this is normal, comparison of median and ulnar nerve sensory or mixed conduction over a 7 to $8-\mathrm{cm}$ distance across the wrist, comparison of median with radial or ulnar nerve sensory conduction across the wrist, or comparison of median nerve sensory or mixed conduction in the carpal tunnel with that in the forearm. Median nerve distal motor latency was not considered as standard.

A standardized self-administered hand symptom diagram has been introduced as a diagnostic aid in CTS both in epidemiologic and clinical setting [6,7]. The reliability and performance of the hand diagram have not previously been evaluated in population-based studies.

We used nerve conduction tests in a population-based study of CTS [8]. The main purpose of the present analysis was to compare the diagnostic accuracy of various tests and determine the properties of the most accurate test when used in population-based CTS. A secondary objective was to evaluate the performance of the standardized hand symptom diagram.

\section{Methods}

A sample of 3,000 persons, aged 25-74 years, was randomly selected from the population register of the 170,000 inhabitants of a southern Swedish region. The Ethics Committee at Lund University's Medical Faculty approved the study. A questionnaire inquiring about demographics, general health, medical history, as well as the presence of numbness, tingling or pain in any part of the body was mailed to the 3,000 persons. Two reminders were mailed when necessary. All responders who reported numbness and/or tingling in the median nerve distribution in the hands at least twice weekly during the preceding four weeks were invited to undergo clinical examination and nerve conduction tests. Control persons randomly selected among the responders who did not report any symptoms in the hands, systemic disease, previous wrist fracture or previous surgery for CTS were also invited for examinations.

The symptomatic persons who attended the examination completed a standardized hand diagram on which they marked pain, numbness, tingling and loss of sensation (each symptom was marked on 2 diagrams depicting the dorsal and volar aspects of the hand and upper extremity); the diagram classifies CTS into four categories (classic, probable, possible, or unlikely) [7]. The symptomatic persons were then examined by the same hand surgeon who, based only on the history and clinical findings, diagnosed each person as having clinically certain CTS or uncertain CTS. The diagnosis of clinically certain CTS required the presence of recurring nighttime or activity-related numbness or tingling involving the palmar aspects of at least two radial fingers. The presence of positive Tinel or Phalen's test and of median nerve sensory or motor deficit was considered supportive of the diagnosis. All hands on which surgery for CTS had been performed were excluded. The control persons were examined to verify the absence of hand symptoms.

Following the clinical examination the symptomatic and asymptomatic persons underwent bilateral nerve conduction tests performed by three blinded technicians using the Viking IV apparatus (Nicolet, Madison, WI). Hands with skin temperature below $30^{\circ} \mathrm{C}$ were warmed. The nerve conduction tests were done using the Kimura technique [9] that utilizes anatomical landmarks and included measurements of median nerve distal motor latency, long finger-wrist sensory latency, and sensory conduction velocity in the forearm, wrist-palm, and palm-digit segments, as well as ulnar nerve small finger-wrist sensory latency.

The hand symptom diagrams were rated independently by two blinded orthopedic surgeons according to the specified criteria [7]; both surgeons were blinded to the clinical and electrophysiological data. A random sample of 63 diagrams was selected and given to each of the two surgeons for repeated rating.

\section{Statistical analysis}

The mean values and 95\% confidence intervals for the neurographic variables were calculated for the right hands and the values for persons diagnosed with clinically certain CTS were compared with those for two groups 
(asymptomatic controls only and asymptomatic controls combined with the symptomatic persons diagnosed with clinically uncertain CTS). The Mann-Whitney U test was used to compare these groups. Analysis with receiver operating characteristic (ROC) curves $[10,11]$ was used to compare the diagnostic power of the nerve conduction tests performed in discriminating the persons with clinically certain CTS from the asymptomatic controls. An ROC curve (a graph of "sensitivity" and " 1 minus specificity" for different cutoff values) is used to compare the accuracy of diagnostic tests; larger area under curve indicates higher accuracy [11]. The tests compared were median nerve distal motor latency, long finger-wrist sensory latency, and wrist-palm sensory conduction velocity, as well as median-ulnar sensory latency difference and comparison of median nerve sensory conduction in the carpal tunnel with that in the forearm. The area under each test's ROC curve and the statistical significance of the difference between the ROC curves were determined using the ROCKIT software [12]. The ROC analysis comprised all symptomatic and control hands. The test shown to have the highest diagnostic accuracy was then further analyzed with regard to sensitivity, specificity and predictive values using the optimal cutoff value (value that minimizes sum of false positives and false negatives) as shown on the ROC curve.

The proportion of diagrams classified as "classic/probable CTS" by at least one rater and those classified as "possible/ unlikely CTS" by both raters were calculated for the persons diagnosed with clinically certain CTS and for those with clinically uncertain CTS. Assessment of interrater and intrarater reliability for the hand diagram was done using the kappa coefficient.

\section{Results}

\section{Survey}

Of the 2,466 survey responders, 354 reported numbness and/or tingling in the median nerve distribution in the hands.

\section{Clinical examination}

Of the 287 (81\%) symptomatic responders who attended the clinical examination, 25 were excluded because of previous surgery for CTS in the symptomatic hand, absence of symptoms in the median nerve distribution (10 persons), or unwillingness to undergo nerve conduction tests. Of the remaining 262 symptomatic persons, 94 (67\% women) were diagnosed by the examining surgeon as having clinically certain CTS. The mean age of these persons was 52 (SD, 13) years. Symptoms were bilateral in 59 persons $(63 \%)$ and right-sided in 27 persons. The 168 persons (65\% women) who were diagnosed with clinically uncertain CTS had a mean age of 53 (SD, 13) years. Symptoms were bilateral in 104 persons (62\%) and right-sided in 51 persons. Of the 134 asymptomatic responders who attended the clinical examination, 9 were excluded ( 7 had numbness and tingling in the hand) and the remaining 125 (55\% women) underwent nerve conduction tests. The mean age of these persons was 51 (SD, 14) years.

\section{Hand diagram}

The hand symptom diagram was completed by 92 of the 94 persons diagnosed with clinically certain CTS; 83 (90\%) were classified as "classic/probable CTS" (82 by both raters) and 9 as "possible/unlikely CTS". The diagram was completed by 163 of the 168 persons with clinically uncertain CTS; 105 (64\%) were classified as "classic/ probable CTS" (99 by both raters) and 57 (36\%) as "possible/unlikely CTS". Thus, using the clinical diagnosis of CTS as criterion standard the hand diagram had at best $90 \%$ sensitivity and 39\% specificity among this population-based sample of persons with numbness and tingling in the hand.

High interrater reliability (kappa, 0.89) and intrarater reliability (kappa, 0.92 and 0.96) was found.

\section{Nerve conduction tests}

The 86 persons with clinically certain CTS involving the right hand had significantly worse median nerve latencies and conduction velocities than the controls, but ulnar latencies were similar (Table 1). Analysis with ROC curves showed no statistically significant differences between median nerve distal motor latency, digit-wrist sensory latency, wrist-palm sensory conduction velocity, and wristpalm/forearm sensory conduction velocity ratio in the power to discriminate the persons with clinically certain CTS from the asymptomatic controls (area under ROC curve, 0.75-0.76). Median-ulnar digit-wrist sensory latency difference demonstrated significantly higher diagnostic accuracy with area under ROC curve of 0.80 (Table 2). Analyses restricted to women or men or to persons with "classic/probable CTS" diagrams gave similar results.

Using the optimal cutoff value of $0.8 \mathrm{~ms}$ for abnormality, median-ulnar nerve digit-wrist sensory latency difference had a sensitivity of $70 \%$, a specificity of $82 \%$, a positive predictive value of $19 \%$ and a negative predictive value of 98\% (Table 3).

\section{Discussion}

The present study is the first population-based investigation that compared the performance of various nerve conduction tests in the diagnosis of CTS. The results revealed that measurement of median nerve distal motor latency, digit-wrist sensory latency, and wrist-palm sensory conduction velocity as well as comparison of sensory conduction velocity in the carpal tunnel with that in the forearm 
Table I: Results of the nerve conduction tests (right hands) in the persons with carpal tunnel syndrome (CTS) and the controls

\begin{tabular}{|c|c|c|c|}
\hline $\begin{array}{l}\text { Nerve conduction test, mean }(95 \% \\
\text { confidence interval) }\end{array}$ & Clinically certain CTS $(n=86)$ & Symptomatic Controls $(n=155)$ & Asymptomatic Controls $(n=124)^{*}$ \\
\hline $\begin{array}{l}\text { Median nerve distal motor latency } \\
(\mathrm{ms})\end{array}$ & $4.2(4.0-4.4)^{\dagger}$ & $3.6(3.5-3.7)$ & $3.4(3.3-3.5)$ \\
\hline $\begin{array}{l}\text { Median nerve digit 3-wrist sensory } \\
\text { latency }(\mathrm{ms})^{\ddagger}\end{array}$ & $3.6(3.4-3.8)^{\dagger}$ & $3.0(2.9-3.1)$ & $2.9(2.8-3.0)$ \\
\hline $\begin{array}{l}\text { Median nerve wrist-palm sensory con- } \\
\text { duction velocity }(\mathrm{m} / \mathrm{s})^{\ddagger}\end{array}$ & $39.2(36.5-41.9)^{\dagger}$ & $48.3(46.8-49.8)$ & $50.7(49.4-52.0)$ \\
\hline Ulnar nerve distal motor latency (ms) & $2.7(2.6-2.7)$ & $2.7(2.6-2.7)$ & $2.8(2.7-2.8)$ \\
\hline $\begin{array}{l}\text { Ulnar nerve digit } 5 \text {-wrist sensory } \\
\text { latency (ms) }\end{array}$ & $2.3(2.2-2.3)$ & $2.3(2.3-2.4)$ & $2.3(2.3-2.4)$ \\
\hline $\begin{array}{l}\text { Median-ulnar nerve sensory latency } \\
\text { difference (ms) }\end{array}$ & $1.3(1.1-1.5)^{\dagger}$ & $0.72(0.65-0.79)$ & $0.57(0.52-0.62)$ \\
\hline
\end{tabular}

* One of the 125 controls did not undergo testing on the right hand because of previous laceration. $\dagger$ Significantly worse than in the controls $(\mathrm{p}<$ $0.00 \mathrm{I}$ ). $¥$ Absent sensory responses in 3 persons with CTS (for both tests in 2 persons) and in one symptomatic control (both tests); in calculating means, absent latency values were replaced with the most abnormal value recorded.

Table 2: Receiver operating characteristic (ROC) analysis comparing the diagnostic accuracy of the nerve conduction tests performed on 153 symptomatic hands of persons with clinically certain carpal tunnel syndrome and 247 asymptomatic control hands*

\begin{tabular}{lcc}
\hline Nerve conduction test & Area under ROC curvet & $\begin{array}{c}95 \% \text { confidence interval for difference } \\
\text { (between 2 successive tests) }\end{array}$ \\
\hline Median nerve distal motor latency & 0.75 & $-0.02-0.03$ \\
Median nerve digit 3-wrist sensory latency & 0.76 & $-0.04-0.02$ \\
$\begin{array}{l}\text { Median nerve wrist-palm sensory conduction } \\
\text { velocity }\end{array}$ & 0.76 & $-0.07--0.01$ \\
$\begin{array}{l}\text { Median-ulnar nerve sensory latency difference } \\
\begin{array}{l}\text { Median nerve wrist-palm/forearm sensory con- } \\
\text { duction velocity ratio }\end{array}\end{array}$ & $0.80 \ddagger$ & $0.01-0.08$ \\
\hline
\end{tabular}

* Two of the 125 asymptomatic control persons declined nerve conduction testing on the left hand and one did not undergo testing on the right hand because of previous laceration. $\dagger I$ indicates perfect accuracy and 0.5 indicates accuracy no better than chance. $¥$ Significantly different from the other four tests (two-tailed $p=0.004$ for all comparisons).

Table 3: Diagnostic test properties of digit-wrist median-ulnar sensory latency difference ( 0.8 ms cutoff value for abnormal result) in population-based carpal tunnel syndrome (CTS) with the clinical diagnosis as criterion standard

\begin{tabular}{|c|c|c|c|}
\hline Test property* & $\begin{array}{l}\text { Symptomatic persons with clini- } \\
\text { cally certain CTS }\end{array}$ & Asymptomatic control persons & $\begin{array}{c}\text { Asymptomatic and symptomatic } \\
\text { control persons }\end{array}$ \\
\hline Sensitivity & $70.2(61.0-79.5)(n$ 66/94) & & \\
\hline \multirow[t]{2}{*}{ Specificity } & & $81.6(74.8-88.4)$ & $73.7(68.7-78.8)$ \\
\hline & & $(n$ 102//25) & (n 2/6/293) \\
\hline \multirow{2}{*}{ Positive predictive value (PPV) } & & $18.7(15.0-22.3)$ & $16.2(13.0-19.4)$ \\
\hline & & (n 83/445) & $($ ( $83 / 55 I)$ \\
\hline \multirow[t]{2}{*}{ Negative predictive value (NPV) } & & $97.9(97.2-98.6)$ & $98.0(97.4-98.7)$ \\
\hline & & $(n / 608 / 1643)$ & $(n / 755 / 1790)$ \\
\hline
\end{tabular}

*shown as \% (95\% confidence interval), number of persons from which the rate was derived shown in italic; Sensitivity = true positives/(true positives + false negatives); Specificity = true negatives/(true negatives + false positives); PPV = true positives/(true positives + false positives); NPV = true negatives/(true negatives + false negatives); PPV and NPV were calculated based on the assumption that the same rates of disease or test abnormality found among the samples examined applied to the corresponding groups among all survey responders as described in the text and previously [8]. 
had similar diagnostic power while median-ulnar sensory latency difference demonstrated higher accuracy.

No consensus exists regarding the type and number of nerve conduction tests needed to establish the neurophysiological diagnosis in CTS. Moreover, there is no consensus on the definition of abnormality [13]. In addition to median nerve motor and wrist-digit sensory latency measurements, numerous new tests have been successively introduced to improve the sensitivity of nerve conduction tests [1]. Performing multiple nerve conduction tests on an individual would increase the likelihood of obtaining a false positive result [14]. Measurements of wrist-palm sensory conduction or median-ulnar comparison have been considered superior to distal motor and digit-wrist sensory latency measurements, particularly in detecting patients with mild CTS [15].

The strengths of the present study lie in its populationbased design, the independent clinical and electrophysiological assessments, and the use of ROC curves comparing the overall performance of different diagnostic tests rather than their performance at specific cutoff values. A possible limitation of this study, also shared by almost all previous studies, is the use of the clinical diagnosis of CTS as the criterion standard against which electrodiagnostic tests were assessed. However, no superior criterion standard for the assessment of nerve conduction tests is currently available, and the analysis involved comparison of different tests in the same population. The diagnosis of CTS, which determined the sensitivity of the nerve conduction tests, was made by the examining surgeon before testing and was supported independently by a validated hand diagram. Estimation of specificity was based on the results of nerve conduction tests performed on completely asymptomatic persons randomly selected from a general population. The examining surgeon verified the absence of symptoms in the hands confirming the control persons' responses to the questionnaire.

A discrepancy exists between the results of this study and those of many previous reports regarding the sensitivity and specificity of nerve conduction tests and, in particular, the similar performance of median nerve motor and sensory conduction tests. Although most previous clinical studies have shown sensory conduction tests to be more sensitive than motor conduction tests, this finding has not been consistent [2]. The discrepancy shown might have been caused by several factors such as methodological differences, with the majority of previous studies being based on (1) referred patients with CTS and/or small convenience samples of controls (in those that did include controls) as opposed to random population-based samples, (2) non-blinded as opposed to blinded neurophysiological examinations and interpretations, and (3) compari- sons of sensitivity of different tests in patients using certain cutoff values as opposed to comparison of their overall performance in persons with CTS and controls. The cutoff value would determine the sensitivity of a certain test; for distal motor latency the cutoff values used in different studies have ranged from $3.8 \mathrm{~ms}$ to $4.6 \mathrm{~ms}$ [4]. The requirement, stated in the practice parameter, that each neurophysiological laboratory should have its own "reference values" [5] does not facilitate a standardized assessment of diagnostic tests. Difference in the demographic characteristics and disease severity among the patient populations studied is yet another factor that could explain the differences in the reported sensitivity for nerve conduction tests in CTS. Although the present study, like other studies that have shown moderate sensitivity for nerve conduction tests, may face the frequently stated argument that the "most sensitive" tests were not used, this argument ignores the limitations of these diagnostic tests [13].

The performance of nerve conduction tests in this population-based investigation does not necessarily apply to their performance in the diagnosis of CTS in clinical settings. The sensitivity estimates in the present study may apply to populations that are similar to the sample examined, being representative of a general population with a wide spectrum of disease severity. The results of the tests in this population showed few persons with absent sensory responses (Table 1), more commonly seen in patients with severe CTS.

Although it is recognized that patients with typical CTS might have normal nerve conduction test results, the exact size of this group has not been specifically investigated. In a recent consensus report on CTS, the authors stated that consensus could not be reached regarding the patients with typical symptoms but normal nerve conduction test results [16]. In two prospective clinical studies that used the clinical diagnosis of CTS as well as complete symptom relief after surgery as the criterion standard, the sensitivity of nerve conduction testing was $78 \%$ and $74 \%$, respectively $[17,18]$. The statement made in the practice parameter that the sensitivity of nerve conduction tests exceeds $85 \%$ at a specificity of $95 \%$ [5] appears to lack strong supportive evidence; this can only be determined with a high degree of certainty by performing appropriately designed randomized clinical studies and testing large population random samples of asymptomatic persons [13].

The present study showed a relatively high level of false positive test results $(18 \%)$ for nerve conduction tests using a cutoff value considered optimal according to the ROC analysis. The most common method of estimating specificity has been to perform nerve conduction tests on a group of "normal" persons who in almost all previous 
studies have been recruited among hospital employees or similar convenience samples. The normal values have then been calculated based on the mean values plus 2 to 3 standard deviations recorded in these control groups. Owing to the nature of this method, high specificity is obtained and values of $95 \%$ to $97 \%$ are usually reported [5]. However, when a test the specificity of which had been calculated with this method in a control group is then used in a different population of "normal" persons, the specificity obtained might be markedly worse.

False positive nerve conduction test results in CTS have been reported previously. In a population-based study from England, a mail survey of 1000 persons (age, 18 to 75 years) found that symptoms of numbness, tingling, or pain in at least 2 of the 3 radial fingers were reported by $18 \%$ of the 648 responders [19]. Electrophysiological examination of about half of the symptomatic persons and of 40 randomly selected asymptomatic persons showed median neuropathy (defined as distal motor latency above $4.5 \mathrm{~ms}$ or wrist-digit sensory latency above $3.7 \mathrm{~ms}$ ) in only $18 \%$ of those with symptoms of CTS and in $20 \%$ of the asymptomatic persons [19]. In a study of 50 asymptomatic persons (mean age, 34 years) the specificity of median-ulnar wrist-palm latency difference of $0.5 \mathrm{~ms}$ was reported to be $100 \%$ [14]; the use of the same test and cutoff value in a subsequent study of 1021 job applicants gave false positive test results in $16 \%$ [20]. In another study of 824 workers (mean age, 38 years) the same test and cutoff value also showed abnormal test results in $16 \%$ of the asymptomatic workers (cutoff value of $0.8 \mathrm{~ms}$ also gave false positive test results) [21]. Surprisingly, in the recent extensive literature review [4], on which the updated practice parameter was based, the above-cited studies that have shown a high rate of abnormal nerve conduction test results among completely asymptomatic persons were not included.

In the present study, a cutoff value derived from the standard deviations calculated for the control sample would have yielded a markedly worse sensitivity. In the absence of a universally accepted criterion standard for the diagnosis of CTS and as in other non-perfect diagnostic tests, a cutoff value should be chosen that yields sensitivity and specificity levels that can be considered acceptable for clinical purposes. The results of the ROC analyses showed that, with acceptable levels of specificity, the sensitivity of nerve conduction tests would not be very high. These findings, similar to a previous report [22] support the view that the diagnosis of CTS ought to be clinical, with nerve conduction tests used to provide objective evidence when necessary, and to support the diagnosis in less typical cases. The findings are, however, based on a clinical diagnosis made by an experienced hand surgeon. Because, in clinical practice, physicians with varying experience man- age patients with CTS, the diagnostic role of nerve conduction tests becomes more important.

Relying solely on the clinical examination in making the diagnosis of CTS might lead not only to missing the diagnosis of CTS in some patients but also to incorrect diagnosis and unnecessary surgery in others. On the other hand, relying solely on nerve conduction tests might lead to some patients who actually have CTS being denied surgical treatment because of their normal test results. Despite the limitations of nerve conduction testing, it is the only tool currently available that can provide direct evidence of median neuropathy at the carpal tunnel to strongly support the diagnosis of CTS in symptomatic patients. Nerve conduction testing also provides an assessment of the severity of median neuropathy, which can be helpful in making decisions concerning type of treatment. Furthermore, in clinical research assessing treatment efficacy in CTS, nerve conduction testing can be useful as part of the inclusion criteria to improve the overall diagnostic certainty; this can minimize the risk of misclassification that reduces the study's ability to detect true differences. However, the results of such studies may not be generalized to patients who are diagnosed on clinical grounds only, which is probably more common in clinical practice.

Predictive values of diagnostic tests are dependent upon disease prevalence. The low positive predictive value for nerve conduction testing is not unusual in a disease with a prevalence rate of less than 5\% [8] in the general population. This suggests that nerve conduction tests are not suitable for screening purposes among non-patient populations. The positive predictive value would be much higher when the tests are used on patients presenting with symptoms of numbness and tingling in the hands.

Besides possessing higher diagnostic accuracy than that of isolated median nerve motor and sensory conduction tests, measurement of median-ulnar latency difference has the advantage of being less influenced by factors such as age, height, weight, and hand temperature. It has been suggested that ulnar nerve involvement might be present in CTS as shown by symptoms and sensory loss involving the ulnar fingers and the resolution of these findings after carpal tunnel release $[23,24]$. The similarity of ulnar nerve motor and sensory latencies among the persons with clinically certain CTS and the controls shown in the present study suggests that ulnar nerve conduction abnormalities in association with CTS are uncommon.

The standardized hand symptom diagram [6,7] demonstrated high sensitivity but poor specificity in the diagnosis of CTS among this population-based sample of persons with numbness and tingling in the hand. Consequently, if the diagram is used among populations for the 
purpose of screening for CTS, the final diagnosis ought to be confirmed through a detailed history taken by a physician.

In conclusion, nerve conduction testing has moderate sensitivity and specificity and a low positive predictive value in population-based CTS. Among the various motor and sensory nerve conduction tests, measurement of median-ulnar sensory latency difference appears to have the highest diagnostic accuracy.

\section{Competing interests}

None declared.

\section{Authors' contributions}

IA initiated, designed and coordinated the study, participated in data collection and analysis, and drafted the manuscript; CG, RJ, and EO participated in the design and conduction of the study and the editing of the manuscript. All authors read and approved the final manuscript.

\section{Acknowledgments}

This study was supported by research grants from the Skåne and Kristianstad County Councils. The authors thank Tomas Hammer and Anna Stefansdottir for classifying the hand diagrams.

\section{References}

I. Stevens JC AAEM minimonograph \#26: the electrodiagnosis of carpal tunnel syndrome Muscle Nerve 1997, 20:1477-I486

2. Jablecki CK, Andary MT, So YT, Wilkins DE and Williams FH Literature review of the usefulness of nerve conduction studies and electromyography for the evaluation of patients with carpal tunnel syndrome Muscle Nerve 1993, 16:1392-1414

3. American Academy of Neurology, American Association of Electrodiagnostic Medicine and American Academy of Physical Medicine and Rehabilitation Practice parameter for electrodiagnostic studies in carpal tunnel syndrome: summary statement Neurology 1993, 43:2404-2405

4. Jablecki CK, Andary MT, Floeter MK, Miller RG, Quartly CA, Vennix $M J$ and Wilson JR Second AAEM literature review of the usefulness of nerve conduction studies and needle electromyography for the evaluation of patients with carpal tunnel syndrome Muscle Nerve 2002, 26 (suppI):SI-S53

5. American Association of Electrodiagnostic Medicine, American Academy of Neurology and American Academy of Physical Medicine and Rehabilitation Practice parameter for electrodiagnostic studies in carpal tunnel syndrome: summary statement Muscle Nerve 2002, 25:918-922

6. Katz JN, Stirrat CR, Larson MG, Fossel AH, Eaton HM and Liang MH A self-administered hand symptom diagram for the diagnosis and epidemiologic study of carpal tunnel syndrome J Rheumatol 1990, 17:1495-1498

7. Katz JN and Stirrat CR A self-administered hand diagram for the diagnosis of carpal tunnel syndrome J Hand Surg Am 1990, 15:360-363

8. Atroshi I, Gummesson C, Johnsson R, Ornstein E, Ranstam J and Rosen I Prevalence of carpal tunnel syndrome in a general population JAMA 1999, 282:153-158

9. Kimura J Electrodiagnosis in diseases of nerve and muscle: principles and practice Philadelphia, FA Davis 1989, 105-109

10. Hanley JA and McNeil B] A method of comparing the areas under receiver operating characteristic curves derived from the same cases Radiology 1983, 148:839-843

II. Sackett DL, Haynes RB and Tugwell P Clinical epidemiology: a basic science for clinical medicine Boston, Little, Brown 1991,

12. ROCKIT software: version 0.9B University of Chicago 1998 ,
13. Smith NJ Nerve conduction studies for carpal tunnel syndrome: essential prelude to surgery or unnecessary luxury? J Hand Surg Br 2002, 27:83-85

14. Redmond MD and Rivner MH False positive electrodiagnostic tests in carpal tunnel syndrome Muscle Nerve 1988, I I:51 I-5 I8

15. Jackson DA and Clifford JC Electrodiagnosis of mild carpal tunnel syndrome Arch Phys Med Rehabil 1989, 70:199-204

16. Rempel D, Evanoff B, Amadio PC, de Krom M, Franklin G, Franzblau A, Gray R, Gerr F, Hagberg M, Hales T, Katz JN and Pransky G Consensus criteria for the classification of carpal tunnel syndrome in epidemiologic studies Am J Public Health 1998, 88: I447$|45|$

17. Finsen $\mathrm{V}$ and Russwurm $H$ Neurophysiology not required before surgery for typical carpal tunnel syndrome J Hand Surg Br 200I, 26:6I-64

18. Atroshi I and Johnsson R Evaluation of portable nerve conduction testing in the diagnosis of carpal tunnel syndrome $J$ Hand Surg Am 1996, 21:65I-654

19. Ferry S, Silman AJ, Pritchard T, Keenan J and Croft P The association between different patterns of hand symptoms and objective evidence of median nerve compression: a community-based survey Arthritis Rheum 1998, 41:720-724

20. Bingham RC, Rosecrance JC and Cook TM Prevalence of abnormal median nerve conduction in applicants for industrial jobs Am J Ind Med 1996, 30:355-361

21. Homan MM, Franzblau A, Werner RA, Albers JW, Armstrong TJ and Bromberg MB Agreement between symptom surveys, physical examination procedures and electrodiagnostic findings for the carpal tunnel syndrome Scand J Work Environ Health 1999 , 25: $115-124$

22. Eisen A, Schulzer M, Pant B, MacNeil M, Stewart H, Trueman S and Mak E Receiver operating characteristic curve analysis in the prediction of carpal tunnel syndrome: a model for reporting electrophysiological data Muscle Nerve 1993, 16:787-796

23. Silver MA, Gelberman RH, Gellman $\mathrm{H}$ and Rhoades CE Carpal tunnel syndrome: associated abnormalities in ulnar nerve function and the effect of carpal tunnel release on these abnormalities J Hand Surg Am 1985, 10:710-713

24. Atroshi I, Johnsson R and Ornstein E Endoscopic carpal tunnel release: prospective assessment of $\mathbf{2 5 5}$ consecutive cases J Hand Surg $\mathrm{Br}$ 1997, 22:42-47

\section{Pre-publication history}

The pre-publication history for this paper can be accessed here:

\section{http://www.biomedcentral.com/1471-2474/4/9/prepub}

Publish with Bio Med Central and every scientist can read your work free of charge

"BioMed Central will be the most significant development for disseminating the results of biomedical research in our lifetime. "

Sir Paul Nurse, Cancer Research UK

Your research papers will be:

- available free of charge to the entire biomedical community

- peer reviewed and published immediately upon acceptance

- cited in PubMed and archived on PubMed Central

- yours - you keep the copyright
BioMedcentral 